\title{
The Synthesis of Hybrid Five-Bar Path Generating Mechanisms Using Genetic Algorithms
}

\author{
Mr A.M.Connor, Dr S.S.Douglas \& Dr M.J.Gilmartin
}

Mechanisms \& Machines Research Group, Liverpool John Moores University

\begin{abstract}
This paper presents a methodology for the synthesis of multi-degree of freedom mechanisms using Genetic Algorithms. A Five-Bar mechanism is a 2-DOF system which requires two inputs to fully describe the output motion. In a hybrid mechanism, one of these inputs is supplied by a Constant Velocity (CV) motor and one is supplied by a programmable servo motor. Such configurations can have considerable savings in power consumption when the armature inertia of the servo motor is low when compared to the load inertia.

In the presented synthesis of such mechanisms, the two inputs required are provided by the $C V$ input and the desired position of the end effector. The Genetic Algorithm is used to search for the optimum link lengths and ground point positions to minimise a multi-criteria objective function. The criteria which contribute to the objective function value are the error between the actual path of the end effector and the desired path, the mobility of the mechanism and the RMS value of the servo motor displacements.
\end{abstract}

\subsection{Introduction}

The traditional approach to designing mechanisms is based upon a 'trial an error' approach, where the link lengths and inputs of a mechanism are selected and the output motion then found. If the output motion does not match the desired output motion the process is repeated by proposing an alternative set of link lengths.

Mechanisms synthesis is the result of automating this process. The desired output motion is specified and a mechanism is found which produces this motion. Synthesis can be sub-divided into two parts. Type synthesis is the selection of a mechanism configuration and is usually based on the experience of the machine designer. Some systems [1] have utilised an expert system approach to type synthesis for single-DOF mechanisms.

Dimensional synthesis follows type synthesis and is essentially the process of choosing the link lengths and input motions to give the desired output. Dimensional synthesis is easily achieved by numerical optimisation techniques where the aim is to minimise the error between the actual output motion of a trial mechanism and the specified desired motion.

Genetic Algorithms have several advantages over 'point-to-point' function searches. The broad and parallel nature of the search ensures that local sub-optimal solutions are avoided. Previous work [2,3] has shown that GAs can be used to synthesis simple single-DOF mechanisms. This paper extends the method to the synthesis of hybrid multi-DOF mechanisms with a multi-criteria objective function.

\subsection{Genetic Algorithms}

Both the theory and application of Genetic Algorithms are well documented in the literature [4,5]. The GA used in this study is a simple GA using just the three main operators : selection, crossover and mutation. The selection algorithm uses a 'roulette wheel' method where the probability of selection is proportional to the individuals fitness, or objective function value. This roulette wheel method is normalised with respect to the sum of the objective function values for each generation. This is done because the aim is to minimise the function and it ensures that the solutions with the lowest objective function value (most fit solutions) have the highest probability of selection. This method eliminates the chance of negative objective function values and it is not necessary to use complicated fitness scaling routines.

The control parameter settings for the GA used in this study were;

Population size : 40

Crossover probability : 0.6

Mutation probability : 0.03

No specialised breeding schemes or other techniques were used to enhance the performance of the GA. The aim was to provide a 'benchmark' set of results which can be used to assess the effects of including additional features in the GA, such as sharing, modified mutation operators or search strategies. 


\subsection{The Analysis of Five-Bar Mechanisms}

The Five-Bar mechanism is a 2-DOF mechanism which requires two inputs to fully define the output motion. Figure 1 illustrates such a mechanism.

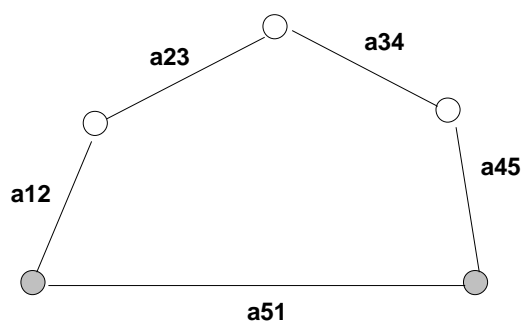

Figure 1 : Five-Bar Mechanism

Each 'stick' in the diagram represents a link between revolute joints. The link $a_{51}$ is the ground link, and so all motions in the mechanism are relative to this fixed datum. The link $\mathrm{a}_{12}$ is the $\mathrm{CV}$ input and so rotates fully around it's ground point. The link $\mathrm{a}_{45}$ is the servo motor input and has a programmable motion.

However, for the sake of the synthesis it is assumed that the motion of the servo motor is not known. To fully analyse the mechanism it is necessary to define an alternative input. To do this it is possible to use the desired position of the end effector. The problem can then be expressed as a search for the mechanism link lengths and the servo motor input displacements which provide the desired end effector motion.

Once the problem has been expressed in these terms, it is possible to describe how an objective function may be constructed so that an appropriate mechanism may be synthesised.

\subsection{Coupler Curve Error}

For the sake of the analysis and synthesis of the Five-Bar mechanism, it is assumed that the end effector is point 3 . This is the revolute joint between links $a_{23}$ and $a_{34}$. The input motion for link $\mathrm{a}_{12}$ is known, and so by defining the position of point 3 it is possible to fully describe the motions in the mechanism. Figure 2 illustrates how the positions of links $\mathrm{a}_{12}$ and $\mathrm{a}_{23}$ can be used to calculate an error score.

A vector is defined from the end of link $\mathrm{a}_{12}$ to the desired position of the end effector. The actual position of this point is then calculated and the error between the two points found. This error is summed around the cycle for the input link and forms the basis of the objective function.

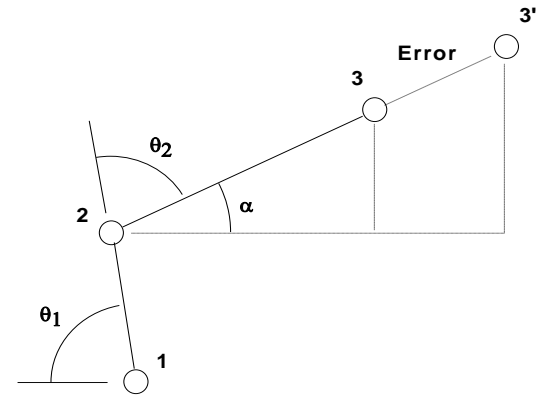

Figure 2 : Calculation of Error

Once the position of the end effector is known, it is possible to calculate the value of $\theta_{2}$ which then makes it possible to calculate the other angles of the mechanism from standard forms. The method used to do this is the Unified Theory [6].

\subsection{Mechanism Mobility}

In section 3.1 it was shown how the two links, $\mathrm{a}_{12}$ and $\mathrm{a}_{23}$, form a dyad which can be used to evaluate the error at a given point for a given input angle. Similarly, the remaining two mobile links, $\mathrm{a}_{34}$ and $\mathrm{a}_{45}$, form another dyad which is used to calculate a penalty function criteria of the overall objective function.

This penalty function is based on the mobility of the mechanism. For a truly mobile mechanism, the dyad formed by the links $\mathrm{a}_{34}$ and $\mathrm{a}_{45}$ should be able to 'close' for all given positions of the CV input crank. This means that the position of point 3, when considered as part of the dyad formed by $\mathrm{a}_{34}$ and $\mathrm{a}_{45}$, should be able to reach the actual position of point 3 defined by the dyad formed by the links $\mathrm{a}_{12}$ and $\mathrm{a}_{23}$.

For each position that this dyad cannot close, the mobility counter is increased. It is important to realise that for each position of the input link, there are two possible closures of the dyad. This is illustrated in Figure 3.

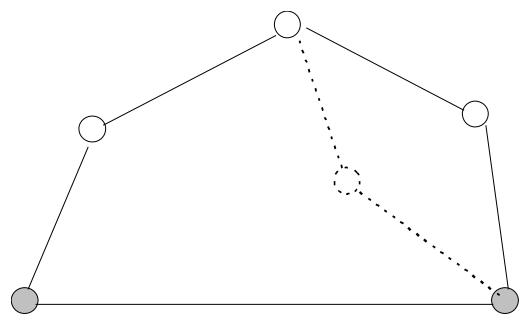

Figure 3 : Multiple Closures of the Mechanism

The implication of this, at this stage, is that for each position of the $\mathrm{CV}$ input link the mobility score may increase by two. The mobility penalty score is summed throughout the cycle and, 
for twenty four precision points, may range between zero and forty eight.

\subsection{Servo Motor Displacements}

Once two inputs to the mechanism are known, in this case $\theta_{1}$ and $\theta_{2}$, it is possible to calculate any other angle in the mechanism. In this case, $\theta_{5}$ is calculated using the standard quadratic expression;

$$
x_{5}=\frac{-b \pm \sqrt{b^{2}-4 a c}}{2 a}
$$

where the coefficients are given by;

$$
\begin{aligned}
& \mathrm{a}=\mathrm{z}_{21}+\mathrm{a}_{45}{ }^{2} / 2-\mathrm{a}_{34}{ }^{2} / 2+\mathrm{a}_{45} \mathrm{y}_{21} \\
& \mathrm{~b}=2 \mathrm{a}_{45} \mathrm{x}_{21} \\
& \mathrm{c}=\mathrm{z}_{21}+\mathrm{a}_{45}{ }^{2} / 2-\mathrm{a}_{34}{ }^{2} / 2-\mathrm{a}_{45} \mathrm{y}_{21}
\end{aligned}
$$

Further expansions can be used to calculate the values of the subscripted $\mathrm{x}, \mathrm{y} \& \mathrm{z}$ terms. However, it suffices to say that these terms are derived from the sine, cosine and sine-cosine laws of the triangles formed by a dyad of two links. This method of analysis is Duffy's Unified Theory method [6].

$\theta_{5}$ is the value of the servo motor displacement calculated from $\mathrm{x}_{5}$ using the following half angle relationship;

$\theta_{5}=2 \tan ^{-1}\left(\mathrm{x}_{5}\right)$

The values for $\theta_{5}$ around the cycle of the mechanism are used to calculate the RMS value of the servo motor displacements. However, the situation is complicated by the presence of multiple closures in the mechanism. For each input position, two values for $\theta_{5}$ can be calculated and the question which arises is 'which value should be chosen?'.

\subsection{Closure Tracking Algorithm}

A simple algorithm has been developed to choose the set of servo motor displacements for a given mechanism. The algorithm is based on the fact that, whilst the overall aim is to minimise the servo motor RMS (and hence minimise the contribution of the servo motor to the bulk motion) it is also desirable to have smooth velocity profiles for the servo motor input.
The algorithm minimises the fluctuation in servo motor velocity in the following way. Given that the mechanism is assumed to start in it's 'open' closure, the algorithm chooses the next displacement angle (from a choice of two) based on the magnitude of the difference in displacement between each possible angle and the starting angle.

For each subsequent point, the algorithm chooses a displacement by considering the difference in magnitude between each possible velocity for the current stage and known velocity of the previous stage. This process is repeated for the cycle of the mechanism.

\subsection{The Objective Function}

The previous sections have outlined how the analysis of a Five-Bar mechanism can be used to calculate three quantities which may be used to construct an objective function for hybrid Five-Bar mechanisms.

Before illustrating how these quantities have been combined to evaluate the objective function value for a given mechanism, it is important to consider what classifies as the "most desirable' mechanism.

The most important factor is a good approximation to the desired output coupler curve. Whilst some errors may be tolerable, depending on the specific application, in this case it is assumed that the magnitude of such errors should be small.

Whilst it is very important for the end effector of the mechanism to follow a desired curve, it is also important that the dyad, formed by the links $\mathrm{a}_{34}$ and $\mathrm{a}_{45}$, is able to close for all input positions. That is, it is important for the mechanism to be mobile.

The final factor that contributes to the objective function is the quality of the servo motor input. In this example, the quality is expressed in terms of the RMS value of the displacements. This is ensures that the servo motor does not contribute a great deal towards the bulk motion of the mechanism, but just 'tweaks' the output to give the desired flexibility.

Each criterion has a weighting factor associated with it, though for this investigation each has been kept at unity. The objective function value is then calculated by the expression;

$\mathrm{obj}=\left(\mathrm{w}_{1} \times\right.$ error $)+\left(\mathrm{w}_{2} \times\right.$ mobility $\left.^{2}\right)+\left(\mathrm{w}_{3} \times \mathrm{RMS}\right)$

\subsection{GA Representation of Solutions}

Now that the objective of the search has been defined, it is possible to explain how a 
solution is represented in GA terms. Each trial mechanism is defined by the position of the two ground points and four link lengths $\left(a_{12}, a_{23}, a_{34} \&\right.$ $\mathrm{a}_{45}$ ). Each link is implicitly constrained by the binary representation to values between 20 and 276 units. This corresponds to a binary string of eight bits for each link.

The ground point positions are defined by $(\mathrm{x}, \mathrm{y})$ co-ordinates within a given constraint envelope. The global $(\mathrm{x}, \mathrm{y})$ co-ordinates of the local origin for the envelopes are specified by the user and each envelope measures 64 by 64 units. This corresponds to a six bit binary string for each co-ordinate value.

As there are four co-ordinates needed to specify the ground points, and the four links, the total length of the binary string defining a given solution is fifty-six bits. The size of the search space is given by $2^{56}-1$. That is, there are $2^{56}-1$ possible solutions which the GA may search.

\subsection{Results}

The GA synthesis method has been tested on a variety of mechanism problems. In each case a known mechanism has been analysed to produce an output curve. This curve has been taken and passed into the synthesis software. The aim of the experiments was to see if the GA could either locate the known mechanism, or a mechanism which produces the same output motion with a more desirable servo motor input.

The graphs shown in Figures 4-7 show the performance of the GA synthesis on one particular problem. The graphs show average results, based on twenty runs of the problem, for the overall objective function and it's components for the best solution in each generation.

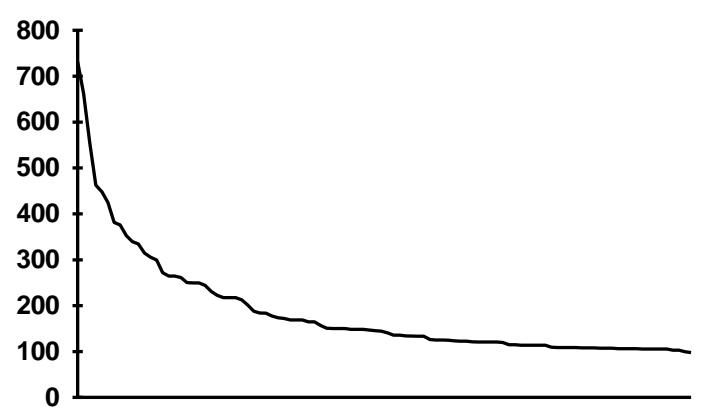

Figure 4 : Average Total Fitness

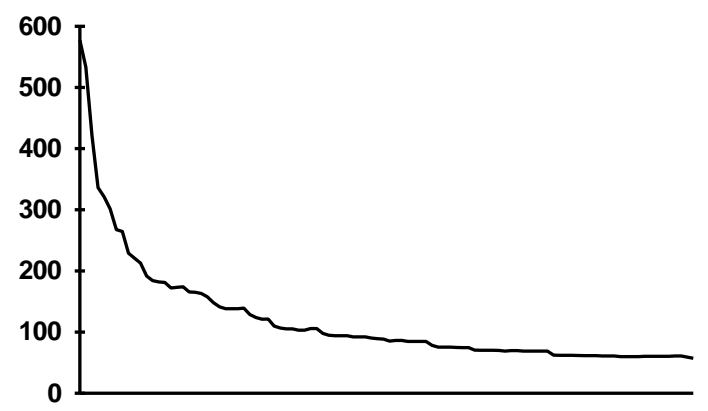

Figure 5 : Average Structural Error

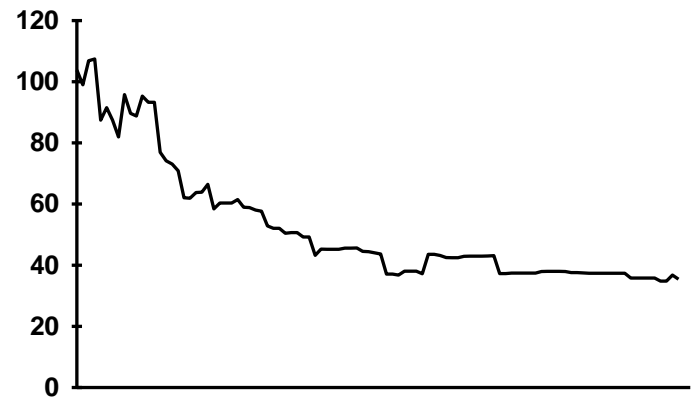

Figure 6 : Average Servo Displacement RMS

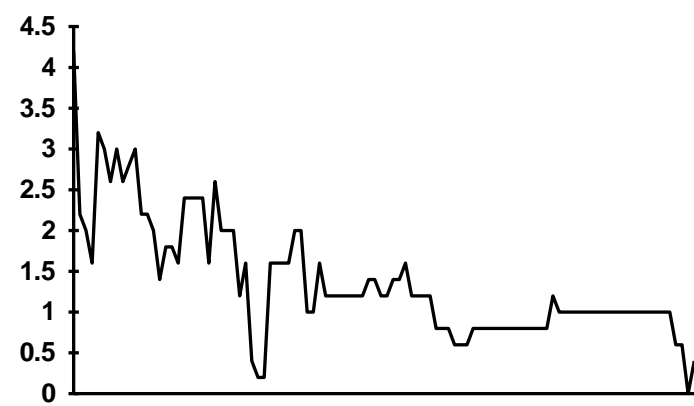

Figure 7 : Average Mechanism Mobility

Of the twenty runs completed in the experiment, the best solution found had the following link lengths and ground point positions.

$$
\begin{array}{ll}
(\mathrm{x}, \mathrm{y})_{1}=-6,0 & \mathrm{a}_{12}=33 \\
(\mathrm{x}, \mathrm{y})_{5}=48,-21 & \mathrm{a}_{23}=56 \\
& \mathrm{a}_{34}=156 \\
& \mathrm{a}_{45}=173
\end{array}
$$

The structural error between the actual and desired curves is 34.42 units, the servo motor displacement RMS value is 26.12 and the mechanism is completely mobile.

Figure 8 shows the actual servo motor displacements of the trial mechanism on the same axes as the servo motor displacements of the known test mechanism. The magnitude of the RMS of the 
displacements is much smaller than that of the test mechanism.

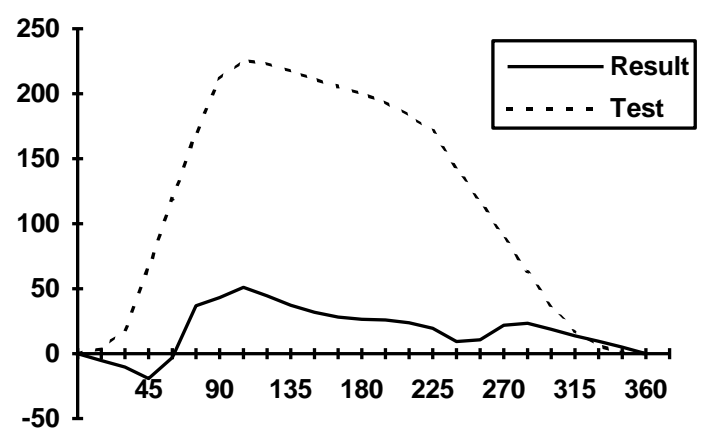

Figure 8 : Servo Motor Displacements

Figure 9 is an $(x, y)$ co-ordinate plot of the output of the end effector of the mechanism, shown by the continuous line. Shown on the same axes are the precision points which define the desired motion of the mechanism.

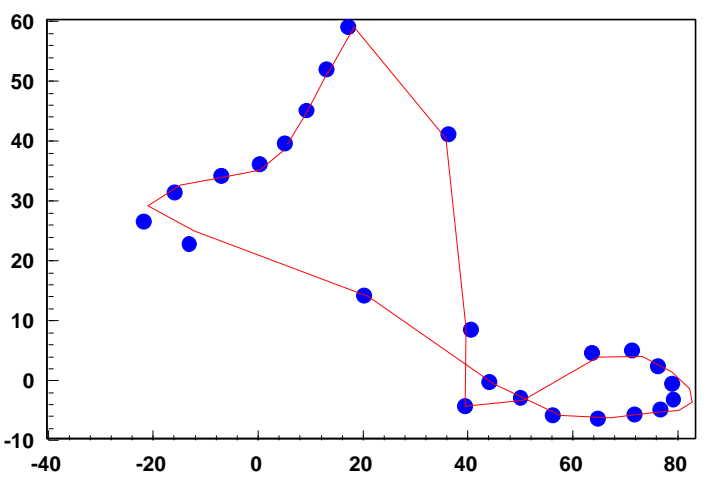

Figure 9 : Mechanism Output Motion

\subsection{Discussion}

Figures 4-9 contain a great deal of information concerning the performance of the GA. Figure 4 is a graph of the average objective function of the best solution of each generation. The graph has the typical characteristics of genetic search, with an initial rapid reduction in fitness followed by a slower, but very definite, improvement. The graph in figure 5, showing the average structural error, is very similar, which is to be expected considering the definition of the objective function (eqn 6).

Figure 6 shows the average servo motor displacement RMS value of the best solution in each generation. The reduction in RMS value is not as smooth as either the error or the overall objective function. Once again this is to be expected as the contribution of the RMS value to the overall objective function is quite small in comparison to the structural error.
Figure 7 shows the average mobility of the best solution. There is only a marginal trend in improvement in mobility. This is due because the results show the average mobility of the best solution of each generation. The best solution is always more likely to be fairly mobile, as well as having a low structural error and so this small trend is to be expected. The fluctuation in the curve can be explained by considering the definition of the objective function. It is possible for a trial mechanism to replace the previous best solution if the difference in structural error of the two mechanisms is great enough to override the mobility penalty.

Figure 8 is a plot of the servo motor displacements of the best solution found in the twenty solution runs. It can be seen from this graph that the servo motor displacements have a much smaller RMS value than for the known test mechanism. However, the displacements fluctuate more than the test mechanism which may give rise to rapid changes in acceleration.

Figure 9 shows the actual output motion of the end effector, plotted as a continuos line, and the precision points used to define the motion. The magnitude of the errors between the actual and desired motions are quite small.

These results show that the performance of the GA is more than adequate for the synthesis of hybrid Five-Bar mechanisms. Despite a huge search space $\left(2^{56}-1\right.$ solutions), the GA has located an acceptable solution after only 4000 trials. The method is quickly locating mechanisms which not only produce the desired motion, but also do so with smaller servo motor displacements.

However, the results have highlighted a short coming in the problem definition. The initial premise of the synthesis would be that smaller servo motor displacements would lead to a mechanism where the bulk of the motion would be provided by the $\mathrm{CV}$ motor. By comparing the best solution from the experiments with the known mechanism the error of this assumption can easily be seen.

The test mechanism that was used to define the desired motion has the following link lengths and ground positions;

$$
\begin{array}{ll}
(\mathrm{x}, \mathrm{y})_{1}=0,0 & \mathrm{a}_{12}=30 \\
(\mathrm{x}, \mathrm{y})_{5}=50,-20 & \mathrm{a}_{23}=50 \\
& \mathrm{a}_{34}=50 \\
& \mathrm{a}_{45}=35
\end{array}
$$

The ground positions and links $\mathrm{a}_{12} \& \mathrm{a}_{23}$ are very similar to the trial mechanism found by the GA. This accounts for the low error between the desired and actual curves. However, the GA has located a mechanism where the links of the closing dyad $\left(\mathrm{a}_{34} \& \mathrm{a}_{45}\right)$ are very much longer than those of 
the test mechanism. These long links produce a greater Cartesian displacement for a given angular displacement. However, the longer links will have a greater mass and so take a greater torque to produce a given angular displacement.

Refinements are obviously needed to the objective function to compromise between the smaller displacements and the greater torque and power requirements and to consider the accelerations of the servo motor as well as the displacements.

\subsection{Conclusions}

The initial results of this study are very promising. The GA is performing as expected and is rapidly locating suitable mechanisms based on the current objective function.

Further will work will be carried out, firstly to refine the definition of the objective function and then to enhance the performance of the GA if required.

\subsection{References}

1. McGarva, J., Rapid Search and Selection of Path Generating Mechanisms from a Library, Mechanisms \& Machine Theory, vol 29, no 2, pp 223-235, 1994.

2. Connor, A.M., The Use of Genetic Algorithms in Optimisation, M.Sc. Dissertation, Liverpool John Moores University, 1994.

3. Connor, A.M., Douglas, S. \& Gilmartin, M., The Kinematic Synthesis of Path Generating Mechanisms Using Genetic Algorithms, Proc. of the Tenth International Conference on Applications of AI in Engineering, Udine, Italy, 1995.

4. Goldberg, D.E., Genetic Algorithms in Search, Optimisation and Machine Learning, Addison-Wesley, 1989.

5. Michalewicz, Z., Genetic Algorithms + Data Structures = Evolution Programs, Springer-Verlag, 1992.

6. Duffy, J., Mechanisms and Robot Manipulators, Arnold, 1980. 